SIGNALLING

\section{Cryptic clues about metastasis}

Many of the signals that regulate tumour growth, metastasis and neovascularization are derived from interactions between cancer cells and the extracellular matrix. Heparan sulphate glycosaminoglycans (HSGAGs) are polysaccharides that are present on the cell surface and in the extracellular matrix, and that bind to and regulate the activities of signalling molecules such as growth factors and cytokines. In the 22 January issue of Proceedings of the National Academy of Sciences, Dongfang Liu et al. show that treatment of mice with two different heparanases - the enzymes that modify HSGAGs — has opposite effects on tumour growth and metastasis.

Heparanase I (Hep-I) cleaves highly sulphated regions of HSGAGs, whereas Hep-III cleaves only the undersulphated region of the polysaccharide chain. In both melanoma and lung carcinoma models, tumour growth was accelerated in mice injected with Hep-I — characterized by increased tumour-cell proliferation, decreased apoptosis and neovascularization. Hep-III treatment of mice, on the other hand, significantly inhibited tumour growth. Hep-III-treated cancer cells were also less invasive in in vitro migration assays, whereas Hep-I treatment increased the ability of cells to migrate by twofold. But does heparanase treatment directly affect growth and metastatic ability of tumour cells, or do these enzymes release bioactive saccharide fragments?

To investigate the role of HSGAG fragments in modulating tumour progression, the authors treated melanoma cells with either Hep-I or Hep-III and isolated the resulting products. Structural analysis of the fragments released by Hep-I or Hep-III treatment confirmed that these were compositionally different and structurally distinct. The different enzymatic products were injected into tumour-bearing mice, and found to recapitulate the biological effects of Hep-I and Hep-III treatment. This indicated that the effects of enzyme treatment were indeed caused by the release of bioactive HSGAG fragments.

But how do these saccharide fragments function? Specific HSGAG structures have previously been shown to bind and modulate fibroblast growth factor 2 (FGF2) activity, and FGF2 signalling has been associated with melanoma progression. So, the authors set out to determine whether these HSGAG fragments also affected FGF2 activity. Hep-III treatment prevented FGF2 stimulation of the extracellular-signal-related kinases Erk1 and Erk2 in melanoma cells, whereas treatment with Hep-I activated Erk1/2 signalling and promoted FGF2mediated proliferation. Exposure of primary tumours to Hep-III (or its products) also inhibited phosphorylation of the FGF receptor-1 (Fgfr1) and focal adhesion kinase activation, whereas Hep-I did not.

These findings indicate that HSGAGs can have either a positive or a negative effect on tumour growth, depending on their composition. Liu et al. conclude that the ability of cells to change the composition or 'signature' of their polysaccharide coat provides them with a mechanism to fine tune the signalling response to the extracellular matrix. Furthermore, a study by Mattias Belting et al. in the 8 January issue of Proceedings of the National Academy of Sciences reports that heparan sulphate proteoglycans are also involved in polyamine internalization, and that glycosaminoglycan (GAG)-deficient tumours grow more slowly in vitro and in vivo. Together, these studies reveal that GAGs might be good targets for anticancer therapeutics.

Kristine Novak

\section{(i) References and links} ORIGINAL RESEARCH PAPER Liu, D. et al. Tumor cell surface heparan sulfate as cryptic promoters or inhibitors of tumour growth and metastasis. Proc. Natl Acad. Sci. USA 99, 568-573 (2002)

FURTHER READING Belting, $M$. et al. Tumor attenuation by combined heparan sulfate and polyamine depletion. Proc. Natl Acad. Sci. USA 99, 371-376 (2002)

\section{WEB SITE}

Ram Sasisekharan's lab:

http://web.mit.edu/tox/sasisekharan

\section{IN BRIEF}

\section{GENOMIC INSTABILITY}

\section{Centrosome amplification drives chromosomal} instability in breast tumor development.

Lingle, W. L. et al. Proc. Natt Acad. Sci. USA 2002 Feb 5; [epub ahead of print]

About $80 \%$ of invasive breast cancers have amplified centrosomes, so does this drive chromosomal instability? By determining chromosomal instability, and centrosome number, size and microtubule nucleation in normal and cancerous breast tissue, Lingle et al. now show that centrosome size and number correlate with chromosomal instability, so centrosome amplification might contribute to tumorigenesis.

\section{MOUSE MODELS}

Genetic analysis of Pten and Ink4a/Arf interactions in the suppression of tumorigenesis in mice.

You, M. J. et al. Proc. Natl Acad. Sci. USA 99, 1455-1460 (2002)

$C D K N 2 A$ - which encodes transcripts of the tumoursuppressor proteins INK4A and ARF - and PTEN are frequently mutated in human cancer, but do they cooperate in tumorigenesis? Cdkn2a $a^{-1-} \mathrm{Pten}^{+/-}$mouse embryonic fibroblasts that are grown in low serum show higher proliferation rates than $\mathrm{Cdkn} 2 a^{-1-} \mathrm{Pten}^{+/+}$cells, and Cdkn2a ${ }^{-1-} \mathrm{Pten}^{+/-}$mice are also more tumour prone and succumb to an increased range of tumour types. The two tumour-suppressor genes therefore seem to cooperate to prevent tumour formation.

\section{GENETICS}

Dominant negative ATM mutations in breast cancer families.

Chenevix-Trench, G. et al. J. Nat/ Cancer Inst. 94, 205-215 (2002)

Do heterozygous mutations in the ataxia-telangiectasia mutated (ATM) tumour suppressor predispose to breast cancer? Studies that indicate that $A T M$ heterozygotes have an increased risk have not been confirmed. But Chenevix-Trench and co-workers now find two ATM mutations that co-segregate with breast cancer in three multiple-case breast cancer families. Both mutations yield a dominant-negative inhibitor of ATM, explaining the dominant nature of these mutations.

\section{DIAGNOSTICS}

\section{Use of proteomic patterns in serum to identify} ovarian cancer.

Petricoin, E. F. et al. Lancet 35, 572-577 (2002)

There is no accurate means, at present, of detecting ovarian cancer in the early stages. This study used mass spectroscopy and algorithms designed to distinguish specific proteomic patterns to analyse blood samples from patients with and without cancer. The authors were able to correctly identify all 50 cases of ovarian cancer, including stage-I cases, and 95\% of the non-cancer controls. This is a great improvement over the ovarian cancer detection technique that is used at present - identifying the tumour marker CA125 combined with ultrasound. 